\title{
Evaluation of the effect of the capitation compensation mechanism among pulmonary tuberculosis patients with a full period of treatment
}

\author{
Xing-Yu Hu and Guang-Ying Gao*
}

\begin{abstract}
Background: PTB is an infectious disease, which not only seriously affects people's health, but also causes a heavier disease economic burden on patients. At present, reform of the medical insurance payment can be an effective method to control medical expenses. Therefore, our study is to explore the compensation mechanism for pulmonary tuberculosis (PTB) patients with a full period of treatment, to alleviate the financial burden of PTB patients and provide a reference and basis for the reform of PTB payment methods in other regions and countries.

Methods: The quantitative data of PTB patients was collected from the first half of 2015 to the first half of 2018 in Dehui Tuberculosis Hospital in Jilin Province, and medical records of PTB patients registered in the first half of 2018 $(n=100)$ from the hospital was randomly selected. Descriptive analysis of these quantitative data summarized the number, cost, medication and compliance. Semi-structured in depth interviews with policymakers and physicians were conducted to understand the impact of interventions and its causes.

Results: After implementation of the compensation mechanism, the number of PTB patient visits in 2018 was increased by $14.2 \%$, average medical costs for outpatients and inpatients were significantly reduced by $31.8 \%$ and $47.0 \%$, respectively, and the auxiliary medication costs was reduced by $36.5 \%$. Moreover, the hospital carried out standardized management of tuberculosis, and the patient compliance was very high, reaching almost $90 \%$.

Conclusions: The capitation compensation mechanism with a full period of treatment was a suitable payment method for PTB, and it is worthy of promotion and experimentation. In addition, the model improved patient compliance and reduced the possibility of drug-resistant PTB. However, due to the short implementation time of the model in the pilot areas, the effect remains to be further observed and demonstrated.
\end{abstract}

Keywords: Pulmonary tuberculosis, Payment method, Capitation, Bundle payment, Evaluation

\section{Background}

The World Health Organization "Global Tuberculosis Report 2019" pointed out that in 2018, an estimated 10 million people worldwide suffered from tuberculosis,

*Correspondence: gaoguangying@yeah.net

School of Public Health, Capital Medical University, No.10 Xitoutiao,

Youanmenwai Street, Fengtai District, Beijing 100069, China and approximately 1.2 million people died. China is the world's second-largest country and has a high burden of tuberculosis [1]. According to the statistics of Chinese Center for Disease Control and Prevention, the number of pulmonary tuberculosis (PTB) cases in China in 2018 was 823 342, accounting for $10.6 \%$ of the total number of infectious disease cases (ranking third in infectious diseases), and the number of PTB deaths was 3149, 
accounting for $13.5 \%$ of the total number of deaths from infectious diseases. PTB has become the second leading cause of death from infectious diseases, after AIDS [2]. Due to the high incidence, high mortality, and wide impact of $\mathrm{PTB}$, it has become a major public health problem that seriously endangers the health of Chinese residents. PTB is also called a "poverty disease". Eighty percent of PTB patients are concentrated in rural areas, and the economic burden of the disease is heavy. PTB has become one of the major diseases that sends individuals back into poverty due to illness, initially causing poverty due to illness and restricting rural social and economic development [3].

From an economic point of view, PTB is a contagious disease, and the spread of PTB has obvious negative external effects. If PTB is not controlled, it not only increases the medical economic cost of patients but also causes social costs that far exceed the individual costs [4]. Since health services have the special nature of supplyside leadership and demand-side passiveness, efforts to reduce the economic burden of PTB patients must start from the source to improve supplier behaviour and effectively control medical expenses. At present, reform of the medical insurance payment mechanism can be an effective method to control medical expenses.

According to the characteristics of PTB itself, based on the clinical path of diagnosis and treatment, this study formulated reasonable service packages, calculated scientific payment standards, and determined a capitation compensation mechanism among PTB patients with a full period of treatment. This change in the compensation mechanism may not only reduce the economic burden of patients' disease but also strengthen the compliance management of PTB patients, which will further help reduce the burden on society. Additionally, this exploration of the compensation mechanism provides a reference for other regions and countries seeking the reform of payment methods.

\section{Methods}

\section{Study sites}

This study relied on the National Health Commission of China-Bill \& Melinda Gates Foundation TB Collaboration project (hereinafter referred to as the "China-Bill \& Melinda Gates project") and selected Dehui City, Jilin Province, China, as the pilot city. The project started in 2016 and ended in 2018 and implemented a capitation compensation mechanism with a full period of treatment.

Therefore, we identified the data from January to July 2018 as the post-reform data and the data from January to July each year in 2015,2016 , and 2017 as the prereform data (Table 1).

\section{Study design}

This study focused on the introduction of a compensation mechanism for PTB with a full period of treatment and used a mixed-methods approach. We combine quantitative analyses of PTB case records from the Hospital Information System (HIS), medical record management system and medical insurance settlement system of Dehui Tuberculosis Hospital, and semi-structured in depth interviews with policymakers and physicians to evaluate its implementation effects.

\section{Data collection}

We searched tuberculosis as the main diagnosis in HIS, medical record management system and medical insurance settlement system of Dehui Tuberculosis Hospital. The time was selected from the first half of 2015 to the first half of 2018, and the search results were all included in the scope of the study.

The dataset contained three basic sets of information: (1) the number of PTB patients, including the number of outpatients and inpatients and the proportion of different types of PTB patients; (2) the cost of PTB patients, including total cost of outpatients and inpatients, average cost, hospital day (HOD), etc. (3) the treatment behaviour of PTB patients, mainly including hepatoprotective drug costs and auxiliary medication costs and their respective proportions.

These records were examined by clinical experts according to the following constraints: (1) the main diagnosis is PTB; (2) the rationality of treatment regimen, defined as whether drugs in the prescription were selected based on PTB disease condition using the reasonable drug treatment by National Diagnostic and Treatment Standards.

In addition to, we conducted a sampling survey and searched all 1116 PTB patients in the first half of 2018 in the medical record management system of Dehui Tuberculosis Hospital. We randomly selected 100 patients based on the patient's hospital number and name as the search criteria, and checked his medical records. The main observation indicators included the number and date of the patient's medication and examination, as well as to estimate the patient's compliance.

\section{Qualitative data}

We conducted semi-structured in-depth interviews to understand the effects of the intervention during the project and the reasons for the problems. In-depth interviews were conducted with the staff of the provincial and prefectural health committees $(n=6)$, the Centers for Disease Control and Prevention $(n=3)$, the Health Insurance Bureau $(n=3)$ and the insurance department of the 


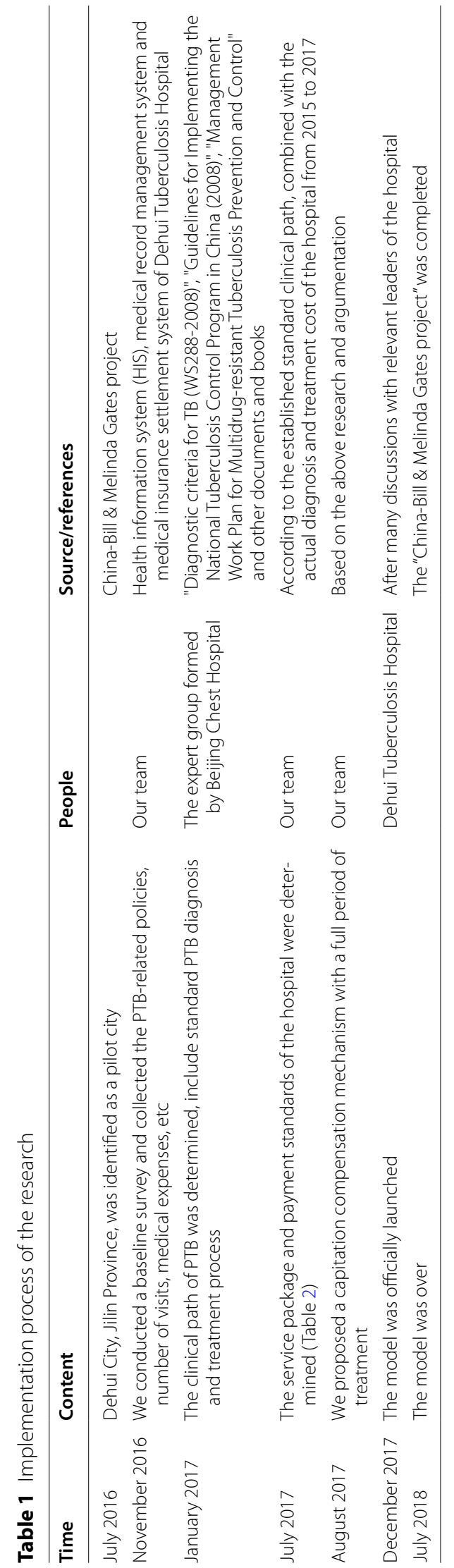


Dehui Infectious Disease Hospital $(n=2)$, to understand the operating effects, the problems and countermeasures that occurred during the operation of the mode. Conducted in-depth interviews with physicians to learn about patients' perception and changes in patients' medical behavior and compliance of the model.

\section{Data analysis}

A descriptive analysis was performed on the patients evaluated in the cohort. Continuous variables were described using mean and the trend of changes over the same period. Categorical variables were summarized using numbers and percentages.

The operation effect of this mode for PTB patients was evaluated in the four areas: patients visits, patient expenses, drug types and costs, and patient compliance.

Indicators were calculated which the data from January to July each year in 2015,2016 , and 2017 as the prereform data, and the data from January to July 2018 as the post-reform data. Compared the cohort data over the same period to see if there is a significant difference between the baseline and the final value.

\section{Mode implementation background and introduction Mode implementation background}

According to the baseline survey, Dehui Tuberculosis Hospital used a project-based payment method before the reform was implemented. In this hospital, PTB has the following characteristics: (1) High hospitalization rate. Since the medical insurance policy of Dehui City does not reimburse the outpatient expenses of tuberculosis patients, people often choose to be directly hospitalized when diagnosed with ordinary PTB, which results in a hospitalization rate of tuberculosis patients as high as $60 \%$. (2) High medical expenses. The hospitalized patients include those with the four types of ordinary initial PTB, ordinary retreatment PTB, PTB pleurisy, and PTB with comorbidities or complications (excluding drug-resistant $\mathrm{PTB}$ ). The average hospitalization costs for these types of PTB in 2016 were Chinese Yuan (CNY) 6608, CNY 6545, CNY 7549, and CNY 8093, respectively. Among them, the proportion of PTB cases with adverse reactions or complications was as high as $16.5 \%$, which is one of the reasons for the high cost of PTB in this hospital. (3) Unreasonable cost structure. Through the analysis of the diagnosis and treatment items of PTB patients in the hospital in 2016, it was found that the diagnosis and treatment items of PTB included drug fees, bacteriological examination fees, imaging examination fees, routine examination fees, diagnosis and treatment service fees, and other expenses. Among them, drug fees and diagnosis and treatment service fees accounted for more than $70.0 \%$, while diagnosis and examination expenses accounted for less than $3.0 \%$, and routine examinations accounted for only $4.9 \%$. Other expenses (mainly liver protection drugs) were as high as $21.3 \%$. This shows that the hospital paid attention to the treatment of PTB but neglected PTB screening and diagnosis and the routine monitoring of the patient's condition. (4) High out-ofpocket rate of tuberculosis patients. The baseline survey showed that the actual compensation ratio for tuberculosis patients in the hospital was $67.5 \%$, and the patient's out-of-pocket rate was much higher than $30.0 \%$ (required by the "China-Bill \& Melinda Gates project").

\section{Model design basis and principles}

1. One of the effective measures to control medical expenses is the reform of medical insurance payment methods. We drew on the idea of capitation and divided PTB into two categories: ordinary PTB and drug-resistant PTB (not involved in the study). Ordinary PTB also included ordinary initial and retreatment PTB, PTB with comorbidities or complications (calculated according to the incidence), and PTB pleurisy. The medical insurance agency presented medical service packages according to the clinical path of each type of PTB, and after reasonable calculation, determined the fixed fee payment standard for each patient (see Table 2) to be paid to the medical service provider (see Fig. 1). This represented a payment system reform suitable for chronic disease management. Its advantages included the promotion of the active control of medical service providers, the strengthening of health education for patients and the standardized management of the entire course of treatment. The disadvantage was the difficulty of determining a reasonable payment standard. Prepayments that were too high or too low had an impact on medical service providers $[5,6]$.

2. The disease characteristics of PTB are presented as follows. First, PTB is a disease that can be cured through standardized treatment. Second, PTB has a relatively clear diagnosis and treatment process. There are standardized treatment plans for PTB in China and around the world, which provide a reference for specific service packages. Finally, if the compliance of PTB patients is improved, the treatment effect can be very good, and the occurrence of drugresistant PTB can be avoided. Therefore, we designed medical insurance payment methods for PTB and provided references for other diseases with relatively clear diagnosis and treatment procedures. 
Table 2 Quota standards for different types of PTB

\begin{tabular}{|c|c|c|c|c|}
\hline Single disease category code & Single disease category name & Treatment & ICD code & $\begin{array}{l}\text { Norm } \\
\text { standard } \\
(\mathrm{CNY})^{\mathrm{ab}}\end{array}$ \\
\hline CD22000101 & Ordinary PTB & Outpatient & A15.300 & 3600 \\
\hline CD22000102 & Ordinary PTB & $\begin{array}{l}\text { Full period (outpatient }+ \text { hospi- } \\
\text { talization) }\end{array}$ & A15.300 & 9000 \\
\hline CD22000201 & PTB pleurisy & Outpatient & A16.500 $\times 004$ & 5000 \\
\hline CD22000202 & PTB pleurisy & $\begin{array}{l}\text { Full period (outpatient }+ \text { hospi- } \\
\text { talization) }\end{array}$ & A16.500 $\times 004$ & 12000 \\
\hline
\end{tabular}

PTB Pulmonary tuberculosis, CNY Chinese Yuan

${ }^{a}$ The standard for the full period of treatment was calculated based on the probability of adverse reactions and complications of PTB

${ }^{b}$ The cost standard is the fixed standard given by medical insurance institutions to medical institutions, and it does not include free first-line anti-tuberculosis drugs invested in by the state or government

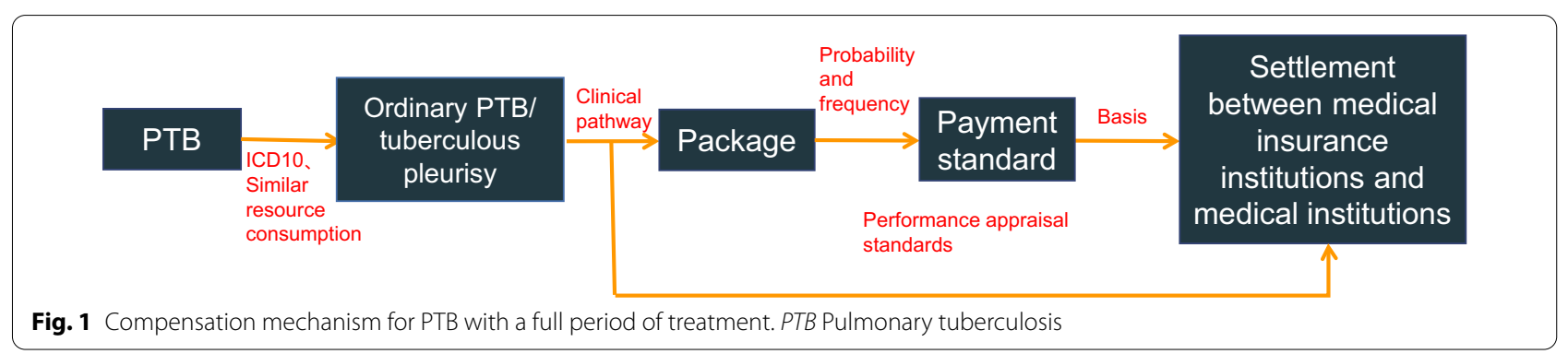

\section{Model introduction}

To alleviate the economic burden of PTB patients and solve the problem of a high hospitalization rate, Dehui Tuberculosis Hospital reformed the medical insurance payment method and designed a capitation compensation mechanism with a full period of treatment. For the policy changes before and after implementation of the reform, refer to Table 3 .

\section{Model features}

The model did not address the reimbursement of hospitalization expenses alone or merely increase the expense reimbursement ratio but rather involved a medical insurance fund payment implementation plan that combined outpatient care and hospitalization. Based on the characteristics of tuberculosis disease, the plan enhanced the importance of prevention based on the principles of prevention first and standardized management to change the medical insurance payment model from the original post-payment model to the pre-payment model. The method of standardized treatment was adopted according to the clinical path, and the surplus funds were returned to the individual, which changed the hospital's operation model from being treatment-oriented to being prevention-oriented.

\section{Results}

Changes in the number of visits for PTB

Dehui City Tuberculosis Hospital treated TB, including $\mathrm{PTB}$, bone $\mathrm{TB}$, renal $\mathrm{TB}, \mathrm{TB}$ meningitis and other $\mathrm{TB}$, but PTB was the main treatment disease, accounting for $90 \%$ of patients in the whole hospital. As noted above, the hospital implemented the compensation mechanism with a full period of treatment in December 2017.

From 2015 to 2017, the number of patient visits to the hospital decreased year by year, but after the implementation of the payment method reform in 2017, the number of visits of PTB patients in 2018 increased by $14.2 \%$, and both outpatients and inpatients increased. Compared with the decrease of $2.9 \%$ in the new incidence of PTB in the region (Jilin Province) in 2018, it can be seen that the implementation of the payment method reform played a certain role in the recovery of the number of PTB patients in the hospital (Table 4).

\section{Changes in the average costs for PTB}

Average medical costs are an important indicator that reflects the situation of medical expenses. After the hospital implemented a full period capitation compensation mechanism at the end of 2017, the average outpatient cost in 2018 was reduced by $31.8 \%$ to almost half of the average cost in 2017, and the average inpatient cost 
Table 3 Policy changes before and after the implementation of the model

\begin{tabular}{|c|c|c|}
\hline Index & Before & After \\
\hline PTB classification & No & $\begin{array}{l}\text { Ordinary PTB/PTB with comorbidities and complications/ } \\
\text { PTB pleurisy }\end{array}$ \\
\hline Medical insurance payment & Fee-for-services & Compensation mechanism with a full period of treatment \\
\hline \multicolumn{3}{|l|}{ Compensation plan } \\
\hline Outpatient & $\begin{array}{l}\text { Special diseases, according to hospitalization com- } \\
\text { pensation }\end{array}$ & Outpatient (according to the norm standard, Table 2) \\
\hline Inpatient & $\begin{array}{l}\text { Deductible is } 600 \text { RMB, The reimbursement rates for } \\
\text { county, township, and village-level medical institu- } \\
\text { tions are } 50 \%, 55 \% \text {, and } 75 \% \text {, respectively } \\
\text { Medical institutions outside the county are not } \\
\text { reimbursed }\end{array}$ & $\begin{array}{l}\text { Full period (according to the norm standard, Table 2) } \\
\text { Cancel the deductible and the non-reimbursable project } \\
\text { expenses outside the medical insurance catalogue } \\
70 \% \text { reimbursement according to the norm standard } \\
\text { Cancellation and project expenses and deductible health } \\
\text { insurance directory can not be reimbursed }\end{array}$ \\
\hline \multicolumn{3}{|l|}{ Billing plan } \\
\hline $\begin{array}{l}\text { Medical institutions and medical } \\
\text { insurance institutions }\end{array}$ & $\begin{array}{l}\text { Hospital compensation amount }=\text { reimbursable } \\
\text { expenses } \times \text { reimbursement ratio } \\
\text { Reimbursable expenses }=\text { total medical } \\
\text { expenses }- \text { non-reimbursable expenses }- \text { deduct- } \\
\text { ible line }\end{array}$ & $\begin{array}{l}\text { Hospital compensation amount }=\text { fixed payment stand- } \\
\text { ard } \times \text { reimbursement ratio }(70 \%) \times \text { number of patients }\end{array}$ \\
\hline Medical institutions and patients & $\begin{array}{l}\text { Patient out-of-pocket expenses }=\text { total medical } \\
\text { expenses }- \text { hospital compensation expenses }\end{array}$ & $\begin{array}{l}\text { Patient out-of-pocket expenses }=\text { total medical } \\
\text { expenses } \times \text { out-of-pocket ratio }(30 \%)\end{array}$ \\
\hline Assessment standard & No & $\begin{array}{l}\text { Whether to implement according to the clinical path, if yes, } \\
\text { all funds are allocated according to the service package } \\
\text { payment standard; if not, } 10 \% \text { of the funds are deducted }\end{array}$ \\
\hline
\end{tabular}

PTB Pulmonary tuberculosis

Table 4 Changes in the number of hospital visits for PTB in the first half of 2015-2018

\begin{tabular}{|c|c|c|c|c|c|c|c|}
\hline \multirow[t]{2}{*}{ Year } & \multirow{2}{*}{$\begin{array}{l}\text { Regional incidence } \\
(1 / 100000)\end{array}$} & \multicolumn{6}{|c|}{ Number of visits for PTB } \\
\hline & & Total & Growth \% & Inpatient & Growth \% & Outpatient & Growth \% \\
\hline First half of 2015 & 56.5 & 1432 & - & 255 & - & 1177 & - \\
\hline First half of 2016 & 49.8 & 1304 & -8.9 & 165 & -35.3 & 1139 & -3.2 \\
\hline First half of 2017 & 50.3 & 977 & -25.1 & 68 & -58.8 & 909 & -20.2 \\
\hline First half of 2018 & 47.4 & 1116 & 14.2 & 78 & 14.7 & 1038 & 14.2 \\
\hline
\end{tabular}

Table 5 Changes in the average costs for PTB in the hospital in the first half of 2015-2018 (Unit: CNY)

\begin{tabular}{lllll}
\hline Year & Inpatient & Growth & Outpatient & Growth \\
\hline First half of 2015 & 527 & - & 7364 & - \\
First half of 2016 & 527 & $0.0 \%$ & 6437 & $-12.6 \%$ \\
First half of 2017 & 523 & $-0.8 \%$ & 6084 & $-5.5 \%$ \\
First half of 2018 & 277 & $-47.0 \%$ & 4152 & $-31.8 \%$ \\
\hline
\end{tabular}

CNY Chinese Yuan, PTB Pulmonary tuberculosis

decreased by $47.0 \%$ year-on-year. The average cost of outpatient and inpatient visits was greatly controlled. This shows that the model had a significant effect on cost control (Table 5).

\section{Changes in PTB treatment behaviour}

Drug therapy is a major method adopted in the clinical treatment of PTB. During the treatment of PTB patients from 2015 to 2017, the cost of adjuvant drugs accounted for a large part of the total medical cost, while the cost of liver-protecting drugs accounted for only approximately $13 \%$. After the implementation of the capitation compensation mechanism with a full period in 2017, the cost of adjuvant drugs for the treatment of PTB was greatly reduced in 2018, and the cost of liver protection drugs increased. This model limited the abuse of auxiliary drugs in the hospital. Further analysis shows that one of the reasons for the substantial reduction in the cost of adjuvant medication was the reduction in the types of adjuvant medications, from an average of 2.8 in 2015 to 1.4 in 2018. After the 
reform, the hospital reduced the use of non-essential auxiliary drugs, and standardized treatment behaviour improved (Table 6).

To address whether the hospital presented sufficient diagnosis and treatment to reduce medical expenses, we researched the average length of hospitalization of PTB patients. The results showed that the average length of hospitalization for PTB patients increased year by year, from 20 days in 2015 to 25 days in 2018. The standard length of hospitalization for the clinical pathway of ordinary PTB was 25 days, and the average length of hospitalization after the reform in 2018 was close to the standard value.

We interviewed hospital administrators and learned that after the reform, when PTB patients were admitted to the hospital, medical staff performed diagnosis and treatment according to the type of PTB and basically followed the service package items set up in accordance with the clinical pathway specifications, so the average length of stay was close to the standard situation. However, we did not further analyse and verify the diagnosis and treatment items of each patient, so this conclusion is for reference only.

\section{Changes in PTB patient compliance}

Compliance can also be called compliance behaviour, which refers to the consistency of a patient's diet, medication, lifestyle, etc. with his or her doctor's advice. It can be divided into three categories: complete compliance, in which the patient shows standardized compliance with his or her doctor's advice and executes it as scheduled; partial compliance, in which compliance cannot be effectively completed for some reason and there are cases in which execution of the doctor's orders is omitted; and non-compliance, in which the changes are made to the doctor's orders without authorization or the patient does not at all follow his or her doctor's advice, buys drugs, stops taking drugs, or abuses drugs [7]. PTB is a disease that can be cured through regular medication. The patient's treatment compliance will directly affect the effect of treatment and the progress of the disease. Therefore, in the process of treating PTB patients, the importance of treatment compliance should be strengthened [8].

We randomly selected 100 patient cases for review in the first half of 2018, and the results showed that patient compliance was very high, reaching almost $90 \%$. Through further interviews with doctors, we found that the previous management model was changed after the payment reform. Not only were diagnosis and treatment conducted in the hospital according to standardized procedures, but discharged patients received follow-up calls by phone, with attention to drug consumption and regular review after discharge to promptly identify patients who stopped taking medication or did not undergo regular review. Then, the hospital contacted patients through the TB prevention network and directed them to the TB prevention institution for treatment. Therefore, the vast majority of patients took their medication and regularly attended their check-up appointments (Table 7).

\section{Discussion}

The capitation compensation mechanism with a full period is suitable for PTB disease and is worthy of promotion and experimentation

After years of clinical practice and exploration, China has developed clear technical specifications for the

Table 7 Compliance of hospital pulmonary tuberculosis patients

\begin{tabular}{lcccc}
\hline Compliance & Take medicine & Proportion & Check & Proportion \\
\hline $\begin{array}{c}\text { Complete } \\
\text { compliance }\end{array}$ & 62 & $62 \%$ & 23 & $23 \%$ \\
$\begin{array}{c}\text { Partial } \\
\quad \text { compliance }\end{array}$ & 35 & $35 \%$ & 62 & $62 \%$ \\
Non-compliance $^{c}$ & 3 & $3 \%$ & 15 & $15 \%$ \\
Total & 100 & 1 & 100 & 1 \\
\hline
\end{tabular}

${ }^{a}$ Complete compliance: Refers to taking medication and checking as prescribed by a doctor for 6 consecutive months and above

${ }^{b}$ Partial compliance: Refers to taking medication and checking as prescribed by a doctor for 1-6 months in a row

'Non-compliance: Refers to not taking medication and checking as prescribed by the doctor

Table 6 Changes in the cost of hepatoprotective drugs and auxiliary drugs in the hospital in the first half of 2015-2018 (Unit: CNY)

\begin{tabular}{lllllllll}
\hline Year & $\begin{array}{l}\text { Total } \\
\text { medical } \\
\text { costs }\end{array}$ & Growth \% & $\begin{array}{l}\text { Hepatoprotective } \\
\text { drug costs (yuan) }\end{array}$ & Growth \% & Percentage & $\begin{array}{l}\text { Auxiliary } \\
\text { medication } \\
\text { costs }\end{array}$ & Growth \% & Percentage \\
\hline First half of 2015 & 4897 & - & 654 & - & 13.4 & 1699 & - & 34.7 \\
First half of 2016 & 5124 & 4.6 & 676 & 3.3 & 13.2 & 1752 & 3.1 & 34.2 \\
First half of 2017 & 4599 & -10.2 & 606 & -10.3 & 13.2 & 2091 & 19.3 & 45.5 \\
First half of 2018 & 4333 & -5.8 & 656 & 8.2 & 15.1 & 1329 & -36.5 & 30.7 \\
\hline
\end{tabular}

CNY Chinese Yuan 
prevention and treatment of PTB. If the patient can be treated in accordance with standardized procedures, the disease can essentially be cured. However, the current medical insurance reimbursement method for PTB covers mainly hospitalization, which leads to the problem of a high tuberculosis hospitalization rate and increases medical insurance fund expenditures. We adopted the capitation compensation mechanism with a full period and combined outpatient treatment and inpatient treatment to promote the effective control of tuberculosis patients in the outpatient stage, prevent patients from undergoing additional hospitalization to obtain medical insurance reimbursement, and use medical insurance funds more efficiently. Additionally, the surplus was used to encourage medical institutions to carry out PTB management and to improve patients' compliance with standardized treatment.

\section{The medical expenses of TB patients have decreased significantly, and the economic burden of the disease has been reduced}

The average cost is an indicator that directly reflects the cost control effect of the payment method. As medical costs increase yearly with economic growth, the change in average cost is also a direct indicator to evaluate the effect of medical cost control. Compared with the situation before the implementation of the project, the average outpatient cost for the hospital decreased by $47.0 \%$, the average inpatient cost decreased by $39.0 \%$, and the average costs of outpatient services and hospitalization were greatly reduced. The payment method reform had a significant effect on cost control. Moreover, the reimbursement ratio of the new plan was $70 \%$, and the patient actually paid only $30 \%$ of all medical expenses, which effectively reduced the patient's economic burden of disease.

\section{Clinical treatment methods have been effectively improved, the rational use of drugs has changed significantly, and the standardized management of diagnosis and treatment behaviour has been improved} In the past, the drug cost of PTB patients accounted for more than $30 \%$ of the medical expenses, the use of hepatoprotective drugs averaged 2-3 species, and there were more than three kinds. The new plan required the standardized management of PTB, especially for the use of non-essential hepatoprotective drugs and auxiliary drugs. After the implementation of the capitation compensation mechanism with a full period of treatment, the cost of auxiliary drugs was greatly reduced, and the types of liver protection drugs gradually decreased and stabilized. The effect of rational drug use began to appear. Additionally, the hospital began to pay attention to and strengthen the awareness of the management with a full period of treatment, minimize hospitalization, reduce costs, and improve the standardized management of PTB.

\section{Patient compliance is improved, which reduces the possibility of the development of drug-resistant PTB} This model effectively improved patient compliance through clinical path management inside the hospital and tracking management outside the hospital. Patients with drug-resistant PTB have two routes of infection. The first route is the development of common tuberculosis. Due to the poor compliance of the patient, treatment is not performed as prescribed by the doctor, which leads to bacterial resistance to PTB drugs. The second route involves infection with drug-resistant strains. The source of infection is drug-resistant patients; thus, the first infection of the patient is drug resistant. Therefore, improving patient compliance is beneficial for reducing the possibility of drug resistance in patients [9]. Because tuberculosis is contagious and has obvious negative external effects, the model that reduced the incidence of tuberculosis and drug-resistant tuberculosis also reduced the burden on society as a whole.

The deficiencies of this research point out that there are two aspects: First, the model selected Dehui Infectious Disease Hospital as a pilot operation for half a year, and the initial effect has been shown. However, due to the short implementation period and single pilot, further long-term promotion in other regions will be needed in the future to verify the effect of this model. Second, the statistical analysis of this study is relatively simple, mainly descriptive analysis without using the model for precise analysis. The above content is also our future research direction.

\section{Conclusions}

This model is worthy of promotion. We used a hospital as a pilot and adopted the capitation compensation mechanism with a full period of treatment. The core idea of this model is to combine prevention and treatment, striving to prevent patients from becoming ill and to keep illnesses minor. This method can improve the standardized treatment and reduce the infection rate of PTB patients, thereby reducing the impact of $\mathrm{PTB}$ on society. The initial operational effect of this model is satisfactory, but the implementation period is short, and further followup observation is needed. Future studies may discuss insufficient diagnosis and treatment services and further refine this model, which may also provide a reference for the reform of PTB payment methods in other regions or countries. 


\section{Abbreviations}

TB: Tuberculosis; PTB: Pulmonary tuberculosis; CNY: Chinese Yuan; AIDS: Acquired immune deficiency syndrome; HIS: Health information system; WS288-2008: Diagnostic criteria for pulmonary tuberculosis (WS288-2008); HOD: Hospital day.

\section{Acknowledgements}

We sincerely thank the Jilin Provincial Center for Disease Control and Prevention and Dehui Tuberculosis Hospital for providing us with data support, the China-Bill \& Melinda Gates Project for providing us with platform support, and all the team members for participating in this study.

\section{Authors' contributions}

$\mathrm{XYH}$ wrote the first draft of the manuscript. GYG and XYH conceived and revised the manuscript. All authors reviewed the final manuscript. All authors read and approved the final manuscript.

\section{Funding}

This study was supported by the National Natural Science Foundation of China, Grant No. 7187040110

The study was supported by the National Health Commission of China-Bill \& Melinda Gates Foundation TB Collaboration project, Grant No. OPP1137180.

\section{Availability of supporting data}

All data generated or analysed during this study were kept confidential by Dehui Tuberculosis Hospital in Jilin Province, China. The datasets are available from the corresponding author on reasonable request.

\section{Declarations}

Ethics approval and consent to participate

This article was based on an analysis of routine surveillance data from China Dehui Tuberculosis Hospital. No personal information was disclosed.

\section{Consent for publication}

Not applicable.

\section{Competing interests}

The authors declare that they have no competing interests.

Received: 26 December 2020 Accepted: 13 May 2021

Published online: 25 May 2021

\section{References}

1. World Health Organization. Global tuberculosis report 2020. Geneva: WHO; 2020. https://www.who.int/tb/publications/global_report/en/. Accessed 3 Nov 2020

2. China CDC. Survey of statutory infectious diseases nationwide; China CDC; 2019. http://www.nhc.gov.cn/jkj/publications/infectiousdiseases_ report/en/. Accessed 20 Apr 2020.

3. The former Bureau of Disease Control and Prevention of the Ministry of Health, the former Medical Administration Department of the Ministry of Health, and the Chinese Center for Disease Control and Prevention. Guidelines for implementing the National Tuberculosis Control Program in China (2008). Beijing: China Union University Press; 2008.

4. Chen W, Liu G, Jiang Q, Li S. Health economics. 4th ed. Beijing: People's Medical Publishing House; 2017.

5. Qiao JJ, Zhu ZF, Jing Q. Discussion on the reform of pay-per-capita payment methods based on case analysis. Chin J Hosp Manag. 2019;05:358-61.

6. Wu J. Thoughts on the per capita payment method for outpatient services in public hospitals. Decis Explor Part 2. 2019;02:89-90.

7. Wu XF, Li YF, Zhang C. Analysis of influencing factors of nucleoside (acid) analogue antiviral therapy compliance in patients with chronic hepatitis B. J Clin Hepatobiliary Dis. 2019;35(10):2194-9.

8. Zhong LM. Research progress on the compliance of antiviral therapy in patients with chronic hepatitis B. Front Med. 2020;10(20):6-8.

9. Tao JQ, Lu H, Wu GQ. Evaluation of the effect of clinical pathways in the inpatient treatment of tuberculosis. J PLA Hosp Manag. 2015;22(7):604-7.
Ready to submit your research? Choose BMC and benefit from:

- fast, convenient online submission

- thorough peer review by experienced researchers in your field

- rapid publication on acceptance

- support for research data, including large and complex data types

- gold Open Access which fosters wider collaboration and increased citations

- maximum visibility for your research: over $100 \mathrm{M}$ website views per year

At BMC, research is always in progress.

Learn more biomedcentral.com/submissions 